\title{
Spin-torque quantization and microwave sensitivity of a nano- sized spin diode
}

\author{
Gleb Demin ${ }^{1,2^{*}}$ and Anatoly Popkov ${ }^{1,2}$ \\ ${ }^{1}$ Moscow Institute of Physics and Technology (MIPT), 141701 Moscow region, Dolgoprudny, Russia \\ ${ }^{2}$ National Research University of Electronic Technology (MIET), 124498 Moscow, Russia
}

\begin{abstract}
Rectification of microwave signal by the spin-torque diode is very promising for its practical applications in microwave imaging. This is due to a very high sensitivity of magnetic tunnel junction under the bias current, which was previously demonstrated in a number of works [1-3]. The decreasing of crosssectional area of the spin-torque diode up to the nano-sized dimensions below $10 \mathrm{~nm}$ allows one to reach high sensitivity without any bias current. Transverse quantization of electron states in the magnetic nanowire based on nano-sized metallic spin valves and magnetic tunnel junctions can create an additional impact not only on the magnetoresistance, but also on the spin-transfer torque in such structures. In this work we present an analysis of the quantization effect of conductance and spin-transfer torques on the microwave sensitivity of nano-sized spin-torque diodes during the reduction of its cross-sectional area. It was found that the magnetoresistance values up to $130 \%$ can be achieved in a magnetic nanowire containing spin-valve diode with the nonmagnetic metal spacer. As a result, the maximum microwave sensitivity of spin-torque diodes based on these structures can be increased several times that opens the way for the further development of highly sensitive microwave detectors.
\end{abstract}

\section{Introduction}

Nowadays much attention in the field of spintronics is paid to the consideration of so-called spin-torque diodes based on magnetic tunnel junctions (MTJ), which have a large magnetoresistive effect and demonstrate very high sensitivity to the microwave signal. Microwave alternating current in MTJ generates dc voltage $V_{d c}=<R(t) I(t)>$ having maximum value at the resonant frequency of spin oscillations induced by the spin-transfer torque [1]. The sensitivity of the spintorque diode $\varepsilon=V_{d c} / P_{i n}$, where $P_{i n}$ is the input microwave power, can exceed the sensitivity of a semiconductor Schottky diode by an order of value in the presence of the bias current [2-4]. High effect of the rectification of the microwave signal in MTJ makes spintorque diode attractive for practical applications in the microwave vision techniques [5]. However, the threshold current density, in the vicinity of which there is a sharp increase in the spin-torque diode sensitivity, is generally high. For that reason there are some problems of the usage of spin-torque diode shifted by bias current. In this connection, it is of interest to consider an alternative way of increasing the sensitivity of a spin-torque diode. This can be realized by reducing its lateral dimensions, since the spin-torque diode sensitivity increases inversely with the area of MTJ [6]. On the other hand, such scaling effects can crucially influence both magnetoresistance and spin-transfer torques in nano-sized region, where the effects of transverse quantization play an important role as it was shown for the conductivity of nano-point magnetic contacts $[7,8]$. In this work we performed an analysis of the microwave sensitivity of magnetic nanosized spin-torque diode based on magnetic tunnel structures depending on the lateral dimensions of its cross-sectional area. We also consider possible effects of quantization of the magnetoresistance and spin-transfer torques in a magnetic nanowire consisting of the metallic spin-valve structure.

\section{Model and basic equations}

\subsection{Charge and spin currents}

We analyze the spin-transfer torque effect in a magnetic nanowire consisting of two semi-infinite ferromagnetic (FM) layers separated by a thin nonmagnetic spacer (S) having the thickness $d_{S}$ and rectangular cross section $a \times b$. Considered trilayer structure «FM-S-FM» of the magnetic nanowire is shown in Fig.1c. The magnetization unit vector $\mathbf{m}_{\mathbf{P}}$ in the reference (left) FM layer is assumed to be fixed, while the magnetization of the free (right) FM layer $\mathbf{m}$ is initially directed at the angle $\theta$ to the direction of magnetization in the reference layer. We use the Sommerfeld model for the description of the ballistic spin transport of free electrons. Within this model the charge and spin fluxes are determined by spinor wave functions $\Psi_{\sigma i}$ of

* Corresponding author: gddemin@gmail.com 
electrons with a given spin polarization according to well-known relations:

$$
\left\{\begin{array}{l}
j_{\sigma i}=-\frac{i \hbar}{2 m_{i^{*}}}\left(\Psi_{\sigma i}^{*} \cdot \frac{\partial \Psi_{\sigma i}}{\partial x}-\Psi_{\sigma i} \cdot \frac{\partial \Psi_{\sigma i}^{*}}{\partial x}\right) \\
j_{\mu, \sigma i}^{s}=\frac{i \hbar}{2 m_{i^{*}}}\left(\left(\frac{\partial \Psi_{\sigma i}^{*}}{\partial x}\right) \sigma_{\mu} \Psi_{\sigma i}-\Psi_{\sigma i}^{*} \sigma_{\mu}\left(\frac{\partial \Psi_{\sigma i}}{\partial x}\right)\right)
\end{array},\right.
$$

where $\hbar$ is the Planck constant, $m_{i^{*}}$ is the effective electron mass in the $i$-th layer, $\sigma_{\mu}$ are the Pauli matrices $(\mu=x, y, z)$. To determine the spinor wave functions $\Psi_{\sigma i}$ in each $i$-th layer $(i=L, S, R)$ for a given spin polarization $\sigma=\uparrow, \downarrow$ of incident electrons, it is necessary to solve the Schrödinger equation:

$-\frac{\hbar^{2}}{m_{i^{*}}} \nabla^{2} \Psi_{\sigma i}(x, y, z)=(E-U(x)) \Psi_{\sigma i}(x, y, z)$,

where $E=E_{\text {oxi }}+E_{\perp m n i}$ is the total energy of the electron including the longitudinal $\left(E_{\sigma x i}\right)$ and transverse $\left(E_{\perp m n i}\right)$ energy terms. In the nano-sized magnetic nanowire the transverse energy is quantized and for the case of its rectangular cross section it can be expressed as $E_{\perp m n i}=\hbar^{2} k_{m n}^{2} / 2 m_{i^{*}}=\beta_{i} E_{m n}$, where $\beta_{i}=m_{e} / m_{i^{*}}$, $k_{m n}=\pi \sqrt{m^{2} / a^{2}+n^{2} / b^{2}}, E_{\sigma x i}=\hbar^{2} k_{\sigma x i}^{2} / .2 m_{i^{*}}, k_{\sigma x i}$ is the spin-dependent longitudinal wave vector in the $i-$ th layer. The spinor wave function $\Psi_{\sigma i}(x, y, z)$ in this case can be presented as the combination of plane waves $\Psi_{\sigma i}(x, y, z)=\psi_{\sigma i}(x) \cdot \Phi_{i}(y, z), \quad$ where $\psi_{\sigma i}(x)=\left(\psi_{\sigma \uparrow_{i}}(x), \psi_{\sigma \downarrow i}(x)\right) \quad$ corresponds to the longitudinal component of the spinor, whereas $\Phi_{i}(y, z)=C_{\varphi} \sin \left(k_{y} y\right) \sin \left(k_{z} z\right)$ is the function of transverse plane wave with the normalization factor $C_{\varphi}=2 / \sqrt{a b}$, where $k_{y}=\pi m / a, k_{z}=\pi n / b \quad$ with quantum numbers $m, n=1,2,3, \ldots$.

We consider the potential profile $U(x)$ in the limit of low bias voltage $\mathrm{eV} \ll E_{F}$ which is shown in Fig. 1a, b for the two types of «FM-S-FM» structure. In general, the $\mathrm{x}$-dependent potential $U(x)$ takes the form:

$$
U(x)=\left\{\begin{array}{l}
-\sigma_{L} \cdot \Delta_{L}+\sigma_{V}^{(-1)}\left(\delta E_{F L, R}+e V\right), x \leq 0 \\
U_{S}, 0<x<d_{S} \\
-\sigma_{R} \cdot \Delta_{R}+\left(\sigma_{V}^{(-1)}-1\right) \delta E_{F L, R}-\sigma_{V}^{(+1)} e V, x \geq d_{S}
\end{array}\right.
$$

where $U_{S}=E_{F}+\varphi_{L}-(\Delta \varphi+e V) x / d_{S}+\sigma_{V}^{(-1)} e V$ in the case of tunnel spacer (Fig. 1a), and $U_{S}=\delta E_{F, N M}$ in the case of metal spacer (Fig. 1b), $\delta E_{F, N M}=E_{F}-E_{F, N M}$, $E_{F, N M}$ is the Fermi level in the metal spacer, $\sigma_{V}^{( \pm 1)}=0.5 \sigma_{V}\left(\sigma_{V} \pm 1\right), \sigma_{V}= \pm 1$ is the sign of the bias voltage $\left(\sigma_{V}=0\right.$ at zero voltage), $e$ is the electron charge, $\delta E_{F L, R}=E_{F R}-E_{F L}, E_{F L(R)}, \Delta_{L(R)}, \varphi_{L(R)}$ are the Fermi level, spin splitting and the work function in the left (right) FM electrode, $E_{F}=E_{F L}+\sigma_{V}^{(-1)} \delta E_{F}$, $\Delta \varphi=\varphi_{L}-\varphi_{R}$, and $\sigma_{L(R)}= \pm 1$ corresponds to the spin direction $\sigma_{L(R)}=\uparrow, \downarrow$ in the left (right) FM layer.

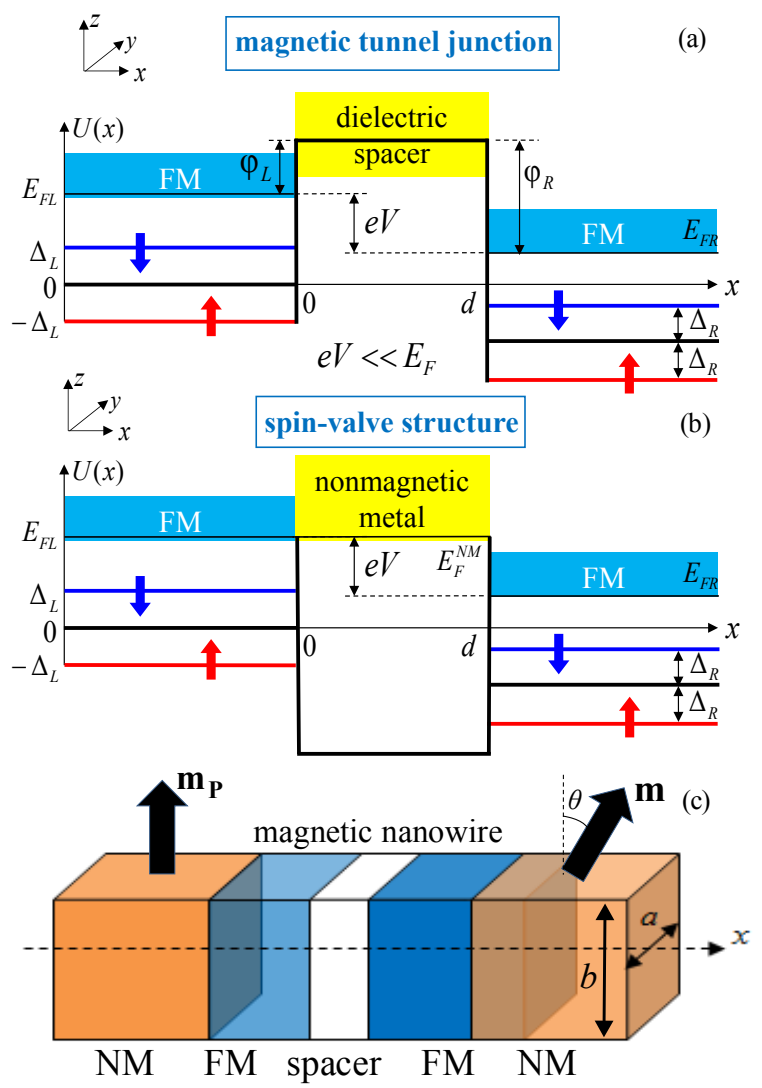

Fig. 1. The schematic potential diagram for the case of (a) magnetic tunnel junction, (b) spin-valve structure. (c) A view of magnetic nanowire with noncollinear magnetization alignment, where NM denotes the «normal metal».

The solution of the quantum-mechanical problem (2) with the potential (3) allows us to find both the charge $\left(<I_{\sigma \sigma^{\prime}(e)}>\right)$ and spin $\left(<I_{\mu, \sigma \sigma^{\prime}(s)}>\right)$ currents in the spin channel $\sigma \leftrightarrow \sigma^{\prime}$ of the proposed structures of magnetic nanowire according to formulas (1) for spin fluxes. Thus, the spin current $\left\langle I_{\mu, \sigma \sigma^{\prime}(s)}>=<I_{\mu, \sigma \sigma^{\prime}(s)}^{L \rightarrow R}>-<I_{\mu, \sigma^{\prime} \sigma(s)}^{R \rightarrow L}>\right.$ can be represented as the difference between the thermodynamically averaged electron spin currents «from the left to the right electrode» $\left(<I_{\mu, \sigma \sigma^{\prime}(s)}^{L \rightarrow R}\right)$, which can be and «from the right to the left electrode» $\left(<I_{\mu, \sigma^{\prime} \sigma(s)}^{R \rightarrow L}>\right)$, which take the following form:

$$
\left\{\begin{array}{l}
\left\langle I_{\mu, \sigma \sigma^{\prime}(s)}^{L \rightarrow R}\right\rangle=\frac{m_{L^{*}}}{2 \pi \hbar^{2}} \cdot \sum_{m, n} \int_{0}^{+\infty} \frac{1}{k_{x L}^{\sigma}} j_{\mu, \sigma \sigma^{\prime} L R}^{(T R)} F_{L R} d E \\
\left.<I_{\mu, \sigma^{\prime} \sigma(e)}^{R \rightarrow L}\right\rangle=\frac{m_{R^{*}}}{2 \pi \hbar^{2}} \cdot \sum_{m, n} \int_{0}^{+\infty} \frac{1}{k_{x R}^{\sigma^{\prime}}} j_{\mu, \sigma^{\prime} \sigma R L}^{(T R)} F_{R L} d E
\end{array},\right.
$$


where $\sigma, \sigma^{\prime}=\uparrow, \downarrow$ is the spin direction of electrons before and after entering the free FM layer, $F_{L R}=f_{L}^{(-1)}\left(1-f_{R}^{(+1)}\right), \quad F_{R L}=f_{R}^{(+1)}\left(1-f_{L}^{(-1)}\right)$, $f_{L(R)}^{(-1)(+1)}=f_{L(R)}\left(E_{V}^{(-1)(+1)}, T\right)$ is the Fermi distribution function in the left (right) FM electrode, $E_{V}^{( \pm 1)}=E+\sigma_{V}^{( \pm 1)} e|V|, \quad T \quad$ is the temperature. $j_{\mu, \sigma \sigma^{\prime} L R}^{(T R)^{\prime}}=j_{\mu, \sigma \sigma^{\prime} L R}^{(T R)}\left(E, E_{m n}\right), \quad j_{\mu, \sigma^{\prime} \sigma R L}^{(T R)}=j_{\mu, \sigma^{\prime} \sigma R L}^{(T R)}\left(E, E_{m n}\right)$ are the transmitted spin fluxes for the $\sigma\left(\sigma^{\prime}\right) \leftrightarrow \sigma^{\prime}(\sigma)$ conduction channel, $E_{m n}$ are quantized energy levels chosen in accordance with the condition $E_{F}-e|V|<E=E_{\sigma\left(\sigma^{\prime}\right) x L(R)}+E_{\perp m n L(R)}<E_{F}$.

\subsection{Quantization of the magnetoresistance and spin-transfer torque}

Thus, the total charge current $\left\langle I_{(e)}\right\rangle$ and the total spin current $\left\langle I_{\mu(s)}\right\rangle$ can be calculated as the sum of corresponding contributions of spin-dependent currents $<I_{\sigma \sigma^{\prime}(e)}>$ and $<I_{\mu, \sigma \sigma^{\prime}(s)}>$ over all possible conducting channels $\sigma\left(\sigma^{\prime}\right) \leftrightarrow \sigma^{\prime}(\sigma)$. Then the conductance of magnetic nanowire can be expressed as $<G_{S}>=\sum_{\sigma, \sigma^{\prime}}<I_{\sigma \sigma^{\prime}(e)}>/ V$ that goes to the Landauer's

formula in the limit of $T \rightarrow 0$ and $V \rightarrow 0$ [9]. In this case, it leads to the following relationships for the charge and spin current:

$$
\left\{\begin{array}{l}
<I_{(e)}>^{T=0 K}=\left.\frac{e^{2} V}{h} \sum_{\sigma, \sigma^{\prime}} \sum_{m, n} P_{T \sigma \sigma^{\prime}(e)}\left(E_{F}, E_{m n}\right)\right|_{E_{m n} \leq E_{F}-U_{\sigma^{\prime}}} \\
<I_{\mu(s)}>^{T=0 K}=\left.\frac{e V}{h} \sum_{\sigma, \sigma^{\prime}} \sum_{m, n} P_{T_{\mu, \sigma \sigma^{\prime}(s)}}\left(E_{F}, E_{m n}\right)\right|_{E_{m n} \leq E_{F}-U_{\sigma^{\prime}}}
\end{array},\right.
$$

where $V$ is the voltage, $P_{T \sigma \sigma^{\prime}(e)}=\left|k_{x R}^{\sigma^{\prime}} / k_{x L}^{\sigma}\right| \cdot\left|T_{\sigma \sigma^{\prime}}\right|^{2}$, $P_{T x, \sigma \sigma^{\prime}(s)}=\operatorname{Re}\left[k_{x R}^{\sigma^{\prime}} / k_{x L}^{\sigma} \mid \cdot\left(T_{\sigma \uparrow}^{*} T_{\sigma \downarrow}\right) e^{-i\left(k_{x R}^{\uparrow}-k_{x R}^{\downarrow}\right) x}\right]$, $P_{T y, \sigma \sigma^{\prime}(s)}=\operatorname{Im}\left[k_{x R}^{\sigma^{\prime}} / k_{x L}^{\sigma} \mid \cdot\left(T_{\sigma \uparrow}^{*} T_{\sigma \downarrow}\right) e^{-i\left(k_{x R}^{\uparrow}-k_{x R}^{\downarrow}\right) x}\right]$, $P_{T z, \sigma \sigma^{\prime}(s)}=\operatorname{Im}\left[i s_{\sigma^{\prime}}\left|k_{x R}^{\sigma^{\prime}} / k_{x L}^{\sigma}\right| \cdot\left|T_{\sigma \sigma^{\prime}}\right|^{2}\right], \quad S_{\sigma^{\prime}=\uparrow, \downarrow}= \pm 1$, $k_{x R}^{\sigma^{\prime}}=k_{x R}^{\sigma^{\prime}}\left(E, E_{m n}\right), \quad T_{\sigma \sigma^{\prime}}=T_{\sigma \sigma^{\prime}}\left(E, E_{m n}\right) \quad$ is the transmission coefficient for the $\sigma \leftrightarrow \sigma^{\prime}$ conduction channel. The amplitudes of in-plane $\left(\tau_{\|}\right)$and out-ofplane $\left(\tau_{\perp}\right)$ spin-transfer torques are proportional to the corresponding spin currents transmitted in the magnetic nanowire:

$$
\left\{\begin{array}{l}
\tau_{\|}=\frac{0.5 \sigma_{V} \hbar}{2}\left\{\left[\sigma_{V}+1\right] \cdot I_{x(s)}(x=d)+\left[\sigma_{V}-1\right]\right. \\
\left.\cdot\left[I_{x(s)}(x=0) \cos \theta-I_{z(s)}(x=0) \sin \theta\right]\right\} \\
\tau_{\perp}=\frac{\sigma_{V} \hbar}{2}\left\{\left[\sigma_{V}+1\right] \cdot I_{y(s)}(x=d)+\left[\sigma_{V}-1\right] \cdot I_{y(s)}(x=0)\right\}
\end{array},\right.
$$

It follows from the expressions (4) - (6) that the quantization of the energy levels for each conduction channel lead both to the conductance quantization $<G_{S}>$ (proportional to $e^{2} / h$ ) and to the quantization of spin-transfer «torquance» $\partial \tau_{\|(\perp)} / \partial V$ (proportional to $e / 4 \pi)$, which should affect the microwave sensitivity of a spin-torque diode.

\subsection{Microwave sensitivity of spin-torque diode based on magnetic nanowire}

In [4] it was shown that the maximum microwave sensitivity $\varepsilon_{M A X}$ of the spin-torque diode in the absence of magnetic field for the case of mutually perpendicular magnetization geometry can be presented:

$\varepsilon_{M A X} \approx \varepsilon_{p} \rho\left(\eta_{\|}+\alpha \eta_{\perp}\right) /\left(\alpha \cdot\left(2 k_{e f f}-k_{b}\right)\right)$,

where $\quad \varepsilon_{p}=v / J_{p} S, \quad v=4 Z_{0} \bar{R} /\left(Z_{0}+\bar{R}\right)^{2} \quad$ is the matching ratio coefficient, $J_{p}=2 e d_{F} M_{S}^{2} / \hbar, Z_{0}$ is the impedance of the line, $S$ is the cross-sectional area of the spin-torque diode, $\bar{R}=2 R_{\uparrow \uparrow}\left(1+\delta_{M R}\right) /\left(2+\delta_{M R}\right)$, $R_{\uparrow \uparrow}=\left(<G_{S}>_{V \rightarrow 0}^{\uparrow \uparrow}\right)^{-1}$ is the diode resistance for the parallel magnetization alignment of FM layers, $d_{F}$ is the thickness of the free FM layer, $M_{S}$ is the saturation magnetization, $\alpha$ is the Gilbert damping factor, $k_{\text {eff }}=2 K_{u} / M_{s}^{2}-4 \pi N_{z}+4 \pi N_{y}, \quad k_{b}=4 \pi\left(N_{y}-N_{x}\right)$, $K_{u}$ is the constant of induced magnetic anisotropy, $N_{x, y, z}$ are the demagnetization factors. In this case $\rho=\delta_{M R} /\left(2+\delta_{M R}\right)$ is the modified value of the magnetoresistance, $\eta_{\|(\perp)}$ is the in-plane (out-of-plane) spin-transfer torque efficiency. It should be noted that for the magnetic nanowire the value of the spin-transfer torque efficiency $\eta_{\|(\perp)}=(2 e / \hbar) \tau_{\|(\perp)}(\theta) /<I_{(e)}(\theta)>, \theta=\pi / 2$, which follows from the definition of the in-plane spintransfer torque $\boldsymbol{\tau}_{\|} \propto m \times m \times m_{P}$ and out-of-plane spintransfer torque $\boldsymbol{\tau}_{\perp} \propto m \times m_{P}$ correspondingly.

\section{Simulation results}

Numerical calculations of the conductance and spintransfer torques in the magnetic nanowire were carried out for the case of its rectangular cross section $(\varsigma=b / a=2)$, where Co was chosen as the material of FM layers with the parameters: $m_{L^{*}}=m_{R^{*}}=m_{e}$, $E_{F L}=E_{F R}=3.24 \mathrm{eV}, \Delta_{L}=\Delta_{R}=1.37 \mathrm{eV}$ (these values correspond to Fermi wave vectors for Co $k_{F}^{\uparrow}=1.1 \mathrm{~A}^{-1}, k_{F}^{\downarrow}=0.7 \mathrm{~A}^{-1}$ estimated for nano-sized magnetic point contact in [8]). The width of the nanowire cross section $a$ was varied in the range from 1 to $3 \mathrm{~nm}$, while the aspect ratio $\varsigma$ was assumed to be 
constant. When considering the magnetic nanowire consisting of a magnetic tunnel junction with $\mathrm{MgO}$ tunnel spacer (the work function $\varphi_{L}=\varphi_{R}=1.2 \mathrm{eV}$, the thickness of $\mathrm{MgO}$ dielectric layer $d_{S}=0.8 \mathrm{~nm}$ ), the effects of the quantization of conductance and spintransfer torques were weakly expressed for the chosen parameters of the tunnel structure. This can be caused by the small transmission probability for various dimensions of the nanowire's cross section. However, in the case of the spin-valve structure (Co-Au-Co) having $d_{S}=0.942 \mathrm{~nm}, E_{F}^{N M}=5.51 \mathrm{eV}$ the quantization of the magnetoresistance $\left.\quad \delta_{M R}=\left(<G_{S}^{\uparrow \uparrow}>/<G_{S}^{\uparrow \downarrow}\right\rangle\right)-1$ was obtained with the increase of its amplitude up to $130 \%$ as it is shown in Fig. 2.
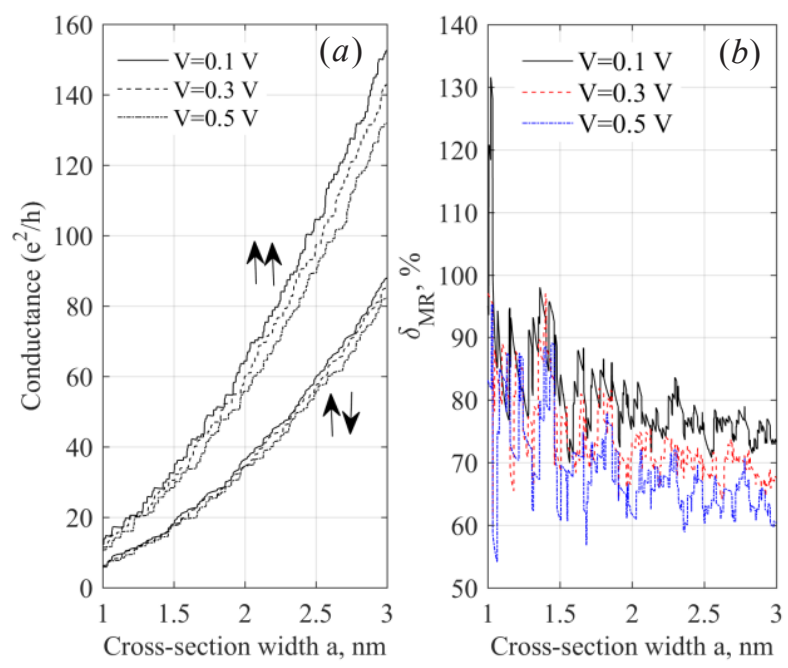

Fig. 2. The dependence of (a) conductance and (b) magnetoresistance of the magnetic nanowire based on spinvalve structure $(\mathrm{Co}-\mathrm{Au}-\mathrm{Co})$ on the cross-section width for the different bias voltages, where $(\uparrow \uparrow(\uparrow \downarrow))$ is the parallel (antiparallel) magnetization alignment of FM layers.

Fig. 3 shows that the quantization of the transverse energy levels in magnetic nanowire also affects the amplitudes of spin-transfer torques. It was found from Fig. 3a that the frequency and the number of quantum jumps will be growing up with increasing the bias voltage. Like in a bulk magnetic tunnel junction, the amplitudes of in-plane $\left(\boldsymbol{\tau}_{\|}\right)$and perpendicular $\left(\boldsymbol{\tau}_{\perp}\right)$ spin-transfer torques have opposite sign in the case of positive bias voltage, but they are additionally quantized due to the confined geometry of magnetic nanowire. The dependence of maximum microwave sensitivity $\varepsilon_{M A X}$ of the spin-torque diode based on the spin-valve structure (Co-Au-Co) from the cross-section width $a$ is shown in Fig. 3b. The following parameters were used: $\varepsilon_{P}=85.5 \mathrm{mV} / \mathrm{mW}, \quad \alpha=0.01, \quad k_{\text {eff }}=0.6, \quad k_{b}=0.3$.

From Fig.3b it can be seen that with the decrease of the characteristic width of the spin-torque diode $a$ the sensitivity of the diode rises and begins to oscillate, starting at approximately $3 \mathrm{~nm}$.
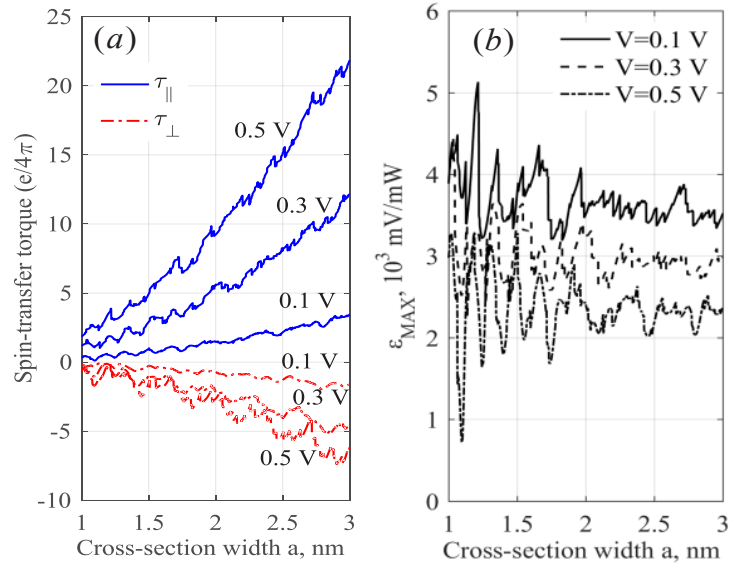

Fig. 3. The quantization of (a) spin-transfer torques and (b) maximum microwave sensitivity in dependence from the crosssection width of magnetic nanowire with the metallic spinvalve structure (Co-Au-Co) for the different bias voltages.

Calculations show that a decrease to lateral sizes less 10 $\mathrm{nm}$ makes it possible to obtain an increase in the microwave sensitivity of the spin-torque diode in the absence of a bias magnetic field by more than two order of value higher than in a nano-pillar spin-torque diode of $100 \mathrm{~nm}$ cross-sectional size. These results can be useful for future development of high-sensitivity microwave detectors. The considered features of the quantization of the spin-transfer torques are very important for the understanding of the physics of magnetoresistive memory elements during its scalability to technological nodes less than $10 \mathrm{~nm}$ [10]. Scaling less than $1 \mathrm{~nm}$ requires new technological and theoretical approaches related to monoatomic physics, which is beyond the scope of this work.

The work was supported by the Russian Science Foundation (project № 16-19-00181).

\section{References}

1. S. Miwa, Nature materials, 13, 50-56 (2014)

2. X. Li, Y. Zhou, P.W.T. Pong, Journal of Nanotechnology, 8347280, 1-11 (2016)

3. B. Fang, M. Carpentieri, X. Hao, Nature Communications, 7, 11259 (2016)

4. A.F. Popkov, N.E. Kulagin, G.D. Demin, Solid State Communications, 248, 140-143 (2016)

5. L. Fu, Y. S. Gui, L. H. Bai, H. Guo, H. AbouRachid, J. Appl. Phys., 117, 213902 (2015)

6. A.F. Popkov, N.E. Kulagin, G.D. Demin, K.A. Zvezdin, Proc.of Universities. Electronics, 22, 101119 (2017)

7. A.K. Zvezdin, A.F. Popkov, J. Exp. Theor. Phys., 71, 209-212 (2000)

8. L.R. Tagirov, B.P. Vodopyanov, B.M. Garipov, J. Magn. Magn. Mater. 258-259, 61-66 (2003)

9. R. Landauer et.al., Phys. Rev. B., 31, 6207 (1985)

10. R. Requist, Nature Nanotech. 11, 499-508 (2016) 